\title{
An Efficient Transport Solver for Tokamak Plasmas *
}

\author{
J.M. Park ${ }^{1}$, M. Murakami ${ }^{1}$, H. St John², L. Lao ${ }^{2}$, M. Chu ${ }^{2}$, and R. Prater ${ }^{2}$ \\ ${ }^{1}$ Oak Ridge National Laboratory, Oak Ridge TN \\ ${ }^{2}$ General Atomics, San Diego CA
}

\begin{abstract}
A simple approach to efficiently solve a coupled set of 1-D diffusion-type transport equations with a stiff transport model for tokamak plasmas is presented based on the 4th order accurate Interpolated Differential Operator scheme along with a nonlinear iteration method derived from a root-finding algorithm. Numerical tests using the Trapped Gyro-Landau-Fluid model show that the presented high order method provides an accurate transport solution using a small number of grid points with robust nonlinear convergence.
\end{abstract}

Keywords: Plasma simulation, Diffusion equation, Transport, Tokamak

E-mail: parkjm@ornl.gov

\footnotetext{
* Notice of Copyright: This manuscript has been authored by UT-Battelle, LLC under Contract No. DE-AC05-00OR22725 with the U.S. Department of Energy. The United States Government retains and the publisher, by accepting the article for publication, acknowledges that the United States Government retains a non-exclusive, paid-up, irrevocable, world-wide license to publish or reproduce the published form of this manuscript, or allow others to do so, for United States Government purposes. The Department of Energy will provide public access to these results of federally sponsored research in accordance with the DOE Public Access Plan (http://energy.gov/downloads/doe-public-access-plan).
}

(C) 2016. This manuscript version is made available under the Elsevier user license http://www.elsevier.com/open-access/userlicense/1.0/ 


\section{Introduction}

Theory-based transport modeling using reduced transport models such as GLF23 [1], MMM95 [2], and TGLF [3] has been widely used to interpret experimental data and guide new experiments for magnetically confined plasmas in Tokamaks.

The reduced models describing turbulent transport resulting from microinstabilities based on drift wave theory have a strong nonlinear dependency on the temperature and density gradients One of the key characteristics of turbulent transport is stiffness, i.e. a sharp increase of turbulent flux as the gradient of temperature increases beyond a certain critical value. Simple numerical schemes like backward Euler or Crank-Nicholson method to integrate the one-dimensional (1-D) flux surface averaged diffusion equations [4] generally suffer from a large numerical oscillation when they are coupled to the stiff transport model. Recently, Newton method has been employed to secure against such numerical instability in a variety of sophistication levels $[5,6,7]$. For example, Globally Convergent Newton Method (GCNM) [8] successfully finds the solution of nonlinear transport equations within a given convergence criteria, but demands relatively long computing time due to additional cost for Jacobian calculation during nonlinear Newton iteration. Although Newton method provides an efficient way to find a solution if a good initial guess is available, it could fail to converge or end up with a local minimum. This paper presents a new simple approach to efficiently solve a coupled set of diffusion equations with the stiff transport model. High order Interpolated Differential Operation (IDO) scheme [9] is employed in conjunction with nonlinear iteration schemes derived from a nonlinear root-finding algorithm to improve the numerical stability. We solve not only the physics variable but also its gradient, especially taking into account that the diffusivity depends strongly on the gradient. This high order scheme allows using a fewer number of grid points for the same accuracy, which helps to minimize calling expensive theory-based transport models like TGLF.

In Section 2 we introduce the model equations and describe the numerical method based on the 4th order accurate IDO scheme. The accuracy and numerical stability are discussed in Section 3 with examples using the TGLF transport model for DIII-D discharges, followed by concluding remarks given in the final section. 


\section{Description of Numerical Scheme}

In this section, we consider a simplified 1-D energy diffusion equation for the magnetically confined toroidal plasma in an axisymmetric Tokamak to describe the numerical scheme.

$$
\frac{3}{2} \frac{\partial}{\partial t}(n T)-\frac{1}{V^{\prime}} \frac{\partial}{\partial \rho}\left(V^{\prime}\left\langle|\nabla \rho|^{2}\right\rangle n \chi \frac{\partial T}{\partial \rho}\right)=S
$$

Here, $n$ is the particle density, $T$ is the temperature of electron or ion species. $V^{\prime}$ is the derivative of the volume enclosed by a flux surface with respect to $\rho$, where $\rho$ is the normalized minor radius proportional to the square root of the toroidal flux. The bracket \langle\rangle denotes the flux surface averaging and $\chi$ is the effective thermal diffusivity. Generally, the theory-based transport model provides the total turbulent flux $Q$ that includes convective and diffusive terms. The effective thermal diffusivity is defined by $Q=\left\langle|\nabla \rho|^{2}\right\rangle n \chi \partial T / \partial \rho$. $S$ is the source term that includes Ohmic heating, ionization, radiation loss, electron-ion heat exchange, and external auxiliary heating such as neutral beam injection and radiofrequency heating. For simplicity, a stationary density $n=n(\rho)$ is assumed to describe the numerical scheme.

We approximate the solution of Eq. (1) using a Hermite interpolation function proposed by Ref. [9]. The interpolation function is constructed by using both the temperature $T_{i}$ and its gradient $T_{i}^{\prime}=\partial T /\left.\partial \rho\right|_{i}$ independently.

$$
T(\rho)=a \rho^{5}+b \rho^{4}+c \rho^{3}+d \rho^{2}+T_{i}^{\prime} \rho+T_{i}
$$

where $i$ denotes the index of grid points $(i=1,2, \ldots, N)$ with an equal interval $\Delta \rho$. The interpolation function covers the area from $i-1$ to $i+1$. The coefficients of the fifthorder polynominal are determined by the four matching conditions of $T(-\Delta \rho)=T_{i-1}$, $T^{\prime}(-\Delta \rho)=T_{i-1}^{\prime}, T(\Delta \rho)=T_{i+1}$ and $T^{\prime}(\Delta \rho)=T_{i+1}^{\prime} ;$

$$
\begin{gathered}
a=-\frac{3}{4 \Delta \rho^{5}}\left(T_{i+1}-T_{i-1}\right)+\frac{1}{4 \Delta \rho^{4}}\left(T_{i+1}^{\prime}+4 T_{i}^{\prime}+T_{i-1}^{\prime}\right) \\
b=-\frac{1}{2 \Delta \rho^{4}}\left(T_{i+1}-2 T_{i}+T_{i-1}\right)+\frac{1}{4 \Delta \rho^{3}}\left(T_{i+1}^{\prime}-T_{i-1}^{\prime}\right) \\
c=\frac{5}{4 \Delta \rho^{3}}\left(T_{i+1}-T_{i-1}\right)-\frac{1}{4 \Delta \rho^{2}}\left(T_{i+1}^{\prime}+8 T_{i}^{\prime}+T_{i-1}^{\prime}\right) \\
d=\frac{1}{\Delta \rho^{2}}\left(T_{i+1}-2 T_{i}+T_{i-1}\right)-\frac{1}{4 \Delta \rho}\left(T_{i+1}^{\prime}-T_{i-1}^{\prime}\right)
\end{gathered}
$$

The higher-order derivatives are obtained by taking the differentials of the interpolation 
function $T(\rho)$.

$$
\begin{gathered}
T_{i}^{\prime \prime}=\left.\frac{\partial^{2} T}{\partial \rho^{2}}\right|_{i}=\frac{2}{\Delta \rho^{2}}\left(T_{i+1}-2 T_{i}+T_{i-1}\right)-\frac{1}{2 \Delta \rho}\left(T_{i+1}^{\prime}-T_{i-1}^{\prime}\right) \\
T_{i}^{\prime \prime \prime}=\left.\frac{\partial^{3} T}{\partial \rho^{3}}\right|_{i}=\frac{15}{2 \Delta \rho^{3}}\left(T_{i+1}-T_{i-1}\right)-\frac{3}{2 \Delta \rho^{2}}\left(T_{i+1}^{\prime}+8 T_{i}^{\prime}+T_{i-1}^{\prime}\right)
\end{gathered}
$$

At the grid point $i=2$, the interpolation function with $a=0$ in Eq. (2) is employed with the boundary condition $T_{1}^{\prime}=0$ and two matching conditions of $T(\Delta \rho)=T_{3}$ and $T^{\prime}(\Delta \rho)=T_{3}^{\prime}$. Similarly, at the grid point $i=N-1, a=0$ is applied with the boundary condition $T_{N}=$ given and two matching conditions of $T(-\Delta \rho)=T_{N-2}$ and $T^{\prime}(-\Delta \rho)=T_{N-2}^{\prime}$.

The equation for time evolution of temperature, Eq. (1), can be written as

$$
\frac{3}{2} n \frac{\partial T}{\partial t}=D T^{\prime \prime}+\left(D^{\prime}+D E\right) T^{\prime}+S
$$

where $D=\left\langle|\nabla \rho|^{2}\right\rangle n \chi$ and $E=\partial / \partial \rho\left(\ln V^{\prime}\right)$. Since the gradient is introduced as an independent variable, we solve the additional equation derived by differentiating Eq. (9).

$$
\frac{3}{2}\left(n \frac{\partial T^{\prime}}{\partial t}+n^{\prime} \frac{\partial T}{\partial t}\right)=D T^{\prime \prime \prime}+\left(2 D^{\prime}+D E\right) T^{\prime \prime}+\left(D^{\prime \prime}+D^{\prime} E+D E^{\prime}\right) T^{\prime}+S^{\prime}
$$

Employing backward Euler scheme for time integration, which is 1st order accurate in time,

$$
\left.\frac{\partial T}{\partial t}\right|_{i}=\frac{T_{i}-T_{0 i}}{\Delta t},\left.\frac{\partial T^{\prime}}{\partial t}\right|_{i}=\frac{T_{i}^{\prime}-T_{0 i}^{\prime}}{\Delta t}
$$

and substituting Eq. (11) into Eq. (9) and Eq. (10), we obtain the finite difference equations for the unknowns $T_{i}$ and $T_{i}^{\prime}$. Here the subscript 0 denotes quantities at the previous time step. The spatial derivatives of the coefficients $D$ and $E$ as well as the source term $S$ are calculated from their cubic spline representation to keep higher order characteristics with a small number of grid points.

The resulting finite difference equations can be written as follows.

$$
\begin{gathered}
\frac{3}{2} \frac{1}{\Delta t} \mathcal{N}\left(\mathcal{T}-\mathcal{T}_{0}\right)-\mathcal{D} \mathcal{T}=\mathcal{S} \\
\mathcal{N}=\left(\begin{array}{cc}
\mathcal{N}_{11} & 0 \\
\mathcal{N}_{21} & \mathcal{N}_{22}
\end{array}\right), \mathcal{D}=\left(\begin{array}{ll}
\mathcal{D}_{11} & \mathcal{D}_{12} \\
\mathcal{D}_{21} & \mathcal{D}_{22}
\end{array}\right),
\end{gathered}
$$

where the $(N-2) \times(N-2)$ matrix $\mathcal{N}_{i j}$ and $\mathcal{D}_{i j}$ are diagonal and tridiagonal respectively 
for the solution vector $\mathcal{T}=\left(T_{2}, \ldots, T_{N-1}, T_{2}^{\prime} \ldots, T_{N-1}^{\prime}\right)^{t} . \mathcal{S}$ is the source vector and the subscript 0 denotes the previous time step. Eq. (12) is strongly nonlinear since $\mathcal{D}$ is a function of $\mathcal{T}, \mathcal{D}=\mathcal{D}(\mathcal{T})$. We apply nonlinear iteration for fully implicit time stepping in order to prevent numerical instability. We solve a linearized equation,

$$
\frac{3}{2} \frac{1}{\Delta t} \mathcal{N}\left(\mathcal{T}^{k}-\mathcal{T}_{0}\right)-\mathcal{D}^{k} \mathcal{T}^{k}=S
$$

with $\mathcal{D}^{k}$ for the next approximation of $\mathcal{D}(\mathcal{T})$ until $\mathcal{D}^{k}$ converges to $\mathcal{D}\left(\mathcal{T}^{k}\right)$. Here the superscript $k$ denotes the nonlinear iteration step.

In this numerical work, we present two relaxation methods. The first method employs Picard type iteration with under-relaxation. $\mathcal{D}^{k}$ is calculated using $\chi^{k}$,

$$
\chi^{k}=\left\{\begin{array}{l}
\alpha \chi\left(T^{\prime k-1}\right)+(1-\alpha) \chi^{k-1} \text { for } k>1 \\
\chi\left(T_{0}^{\prime}\right) \text { for } k=1
\end{array}\right.
$$

where $\alpha$ is the under-relaxation factor. For a steady-state solution $(\partial / \partial t=0)$, this relaxation scheme is equivalent to applying a root-finding algorithm for the solution of $\chi\left(T^{\prime}\right) T^{\prime}=C$ if $\chi$ depends only on $T^{\prime}$, where the constant $C$ does not change with iteration within the discretization error of Eq. (12). Figure 1(a) illustrates the solution procedure for the stiff transport model described in Section 3. The $\chi^{k}$ at the iteration $\mathrm{k}$ converges to the solution of nonlinear equation that is intersection of two lines for $y=\chi\left(T^{\prime}\right)$ and $y=C / T^{\prime}$ if $\chi$ is monotonic function of $T^{\prime}$ and $\alpha$ is small enough.

Generalizing the relaxation scheme on the basis of a root-finding algorithm, the second method employs regula-falsi method that combines features from the secant method and the bisection method. In the secant method, $\chi^{k}$ is evaluated as a solution of $y=C / T$ and the secant line connecting two points $P^{k-1}$ and $P^{k-2}$, where $P^{k}=\left(T^{\prime k}, \chi\left(T^{\prime k}\right)\right)$ as shown in Fig 1(b). In the regula-falsi, the second point, $P^{k-2}$ is replaced by $P^{k-3}$ if

$$
\left(C / T^{\prime k-1}-\chi\left(T^{\prime k-1}\right)\right)\left(C / T^{\prime k-2}-\chi\left(T^{\prime k-2}\right)\right)>0
$$

For time dependent case, $C$ is not constant during the nonlinear iteration and updated using the value at the previous iteration, $C=\chi^{k-1} T^{\prime k-1}$.

Advanced nonlinear scheme could be employed to minimize the number of nonlinear iterations. However, there is a tradeoff between minimizing the nonlinear iteration and calls to the expensive theory-based transport models. For example, the TGLF transport 
model solves 101x101 eigenvalue for 21 toroidal modes at each spatial grid point. Less nonlinear iteration using more sophisticated and complicated nonlinear schemes may not compensate for additional computational costs due to the increased number of TGLF calls per iteration.

\section{Results}

In this section, we show numerical tests with the method described in Section 2 using the FASTRAN (FASt TRANsport) code. FASTRAN is a modular transport solver for a core transport component of the Integrated Plasma Simulator (IPS) [10]. FASTRAN solves a coupled set of transport equations for electron density, electron and ion temperatures, toroidal angular velocity, and poloidal magnetic flux with the input profiles of sources and the metric coefficients provided by the external heating and current drive codes and MHD equilibrium solver. FASTRAN has been used extensively for predictive modeling of DIIID [11, 12, 13], FNSF [14], and ITER [15, 16] with a range of theory-based transport models.

The spatial accuracy of the presented numerical scheme is evaluated in the cylindrical coordinates $(D=n \chi, E=1 / \rho)$ for the sake of simplicity. We compare the numerical solution in steady-state $\partial / \partial t=0$ with the analytic solution, $T=S_{0} /(n \chi)\left(\left(\exp \left(1-\rho^{2}\right)-1\right)\right.$ for $S=S_{0}\left(1-\rho^{2}\right) \exp \left(1-\rho^{2}\right)$ and the constant values of $n$ and $\chi$. Here, the boundary conditions are applied as $T^{\prime}(\rho=0)=0$ and $T(\rho=1)=0$. We estimate a numerical error by the formula

$$
\sigma=\frac{\sum_{i=1}^{N}\left|T_{i}-T_{i, \text { analytic }}\right|}{\sum_{i=1}^{N}\left|T_{i, \text { analytic }}\right|},
$$

where $T_{i, \text { analytic }}$ denotes the analytic solution at the $i$-th grid point. Figure 2 shows the numerical error for the IDO scheme as a function of the grid number for $n=1, \chi=1$, and $S_{0}=4$. The error has a scaling of $(\Delta \rho)^{4}$. Compared with the 2nd order finite difference method (FDM) using

$$
T^{\prime}=\frac{T_{i+1}-T_{i-1}}{\Delta \rho}, T^{\prime \prime}=\frac{T_{i+1}-2 T_{i}+T_{i-1}}{\Delta \rho^{2}}
$$

the IDO scheme requires a much smaller number of grid points for the same accuracy. For example, the IDO solution with $N=11$ is as accurate as the 2nd order FDM solution with $N=101$.

Numerical stability with a stiff transport model is tested using an analytic model diffu- 
sivity,

$$
\chi=\left\{\begin{array}{l}
\chi_{0}+\kappa\left(\left|T^{\prime}\right|-T_{c}^{\prime}\right)^{\gamma} \text { for }\left|T^{\prime}\right|>T_{c}^{\prime}, \\
\chi_{0} \text { for }\left|T^{\prime}\right| \leq T_{c}^{\prime}
\end{array},\right.
$$

where $\kappa$ represents the strength of the stiffness. Figure 3 shows time dependent solution in cylindrical coordinates with $\chi_{0}=1, \kappa=10, \gamma=1$ and $T_{c}^{\prime}=0.5$. Initial condition is $T=0$ for constant $n=1$ and $S=4$. Explicit evaluation of $\chi=\chi\left(T_{0}^{\prime}\right)$ using the temperature gradient at the previous time step, $T_{0}^{\prime}$ results in oscillatory temperature profile both in time and space as shown in Fig 3(b). The implicit time advance with $\chi=\chi\left(T^{\prime}\right)$ is necessary to avoid the numerical instabilities. Figure 3 shows the number of nonlinear iterations to reduce the residual by

$$
R=\frac{\left\|3 /(2 \Delta t) \mathcal{N}\left(\mathcal{T}^{k}-\mathcal{T}_{0}\right)-\mathcal{D}\left(\mathcal{T}^{k}\right) \mathcal{T}^{k}-S\right\|_{2}}{\|\mathcal{S}\|_{2}}<10^{-4} .
$$

The number of iterations is less than 10 both for the under-relaxation method with $\alpha=0.3$ and the regula-falsi method. The steady-state solution $(\partial / \partial t=0)$ with infinite time step $(1 / \Delta t=0)$ also shows robust convergence requiring a small number of iterations, 12 and 9 iterations for the under-relaxation and regula-falsi methods, respectively.

Numerical test with a nearly discontinuous $\chi$ as a function of $T^{\prime}$ also shows an efficient convergence. We employ a discontinuous model diffusivity

$$
\chi=\left\{\begin{array}{l}
\chi_{1} \text { for }\left|T^{\prime}\right| \geq T_{c}^{\prime} \\
\chi_{2} \text { for }\left|T^{\prime}\right|<T_{c}^{\prime}
\end{array}\right.
$$

which is approximated by

$$
\chi=\frac{1}{2}\left(\chi_{1}-\chi_{2}\right)\left(1+\tanh \left(\delta\left(\left|T^{\prime}\right|-T_{c}^{\prime}\right)\right)\right)+\chi_{2} .
$$

The numerical solution with Eq. (21) converges to the solution with Eq. (20) as $\delta$ increases as shown in Fig 4(a). Here, a larger $\delta$ corresponds to a sharper transition of $\chi$ at $\left|T^{\prime}\right|=T_{c}^{\prime}$. The number of nonlinear iterations for the regula-falsi method [Fig 4(b)] increases with $\delta$ but saturates to a finite number of iterations for a steady state solution with $\chi_{1}=2.5$, $\chi_{2}=1$, and $T_{c}^{\prime}=1$.

Figure 5 shows the calculated $T_{e}$ and $T_{i}$ profiles with the TGLF transport model for one of the stationary high beta discharges in DIII-D reported at Ref. [12]. The Chang-Hinton neoclassical diffusivity [17] is added to the TGLF contribution of $\chi$ in Eq. (1). The external 
heating profiles ( $S_{e}$ and $S_{i}$ for electron and ion) from the neutral beam injection (NBI) and electron cyclotron heating (ECH) are calculated by the NUBEAM [18] and TORAY [19] codes, respectively, using the experiment profiles as input. Kinetic EFIT equilibrium reconstruction [20] provides the metric coefficients of $V^{\prime}$ and $\left\langle|\nabla \rho|^{2}\right\rangle$. The time step is set infinite to find a steady-state solution $(\partial d / \partial t=0)$. The boundary condition is given at $\rho=0.8$ with the experimental value as used in the theory-based scenario modeling for core plasma transport [15]. Both the calculated temperature and gradient profiles are smooth in space without artificial oscillations and converge rapidly with the number of grid. The steady-state solution at a small number of grid $(N=11)$ is close to the solution with $N=101$ as shown in Fig 5 .

The nonlinear iteration methods employed in this numerical work show robust convergence with the TGLF transport model for a set of DIII-D high beta discharges. This set of calculations represents a variety of the discharge conditions with large variation in the normalized plasma beta, current and pressure profiles, $T_{e} / T_{i}$ and heat deposition location that change the characteristics of turbulent energy transport for electron and ion. Figure 6 shows the number of iterations for the under-relaxation method as a function of the relaxation parameter, $\alpha$. The iteration number for a steady-state solution decreases with $\alpha$, while the under-relaxation method does not converge at $\alpha>0.3$. There is an optimum $\alpha$ for faster convergence above which the under-relaxation method does not converge. On the other hand, the regula-falsi method does not have a control parameter to adjust and converges within 15 iterations on average, which is slightly higher than the average number of iterations at the optimum $\alpha=0.25$ for the under-relaxation method.

\section{Conclusion}

A simple approach to efficiently solve a coupled set of 1-D diffusion equations with the stiff transport model for tokamak plasmas is presented based on the 4th order accurate IDO scheme. The presented high order method in space provides accurate transport solution with a small number of grids by solving both the physics variable and its spatial gradient. This method employs a simple nonlinear iteration derived by root-finding algorithms, showing robust convergence with a small number of nonlinear iterations for implicit time stepping or finding a steady-state solution. Recent remarkable advances in the theory-based turbulent transport model for tokamak plasmas demands large computational resources when coupled to the 1-D transport solver to predict time evolution of tokamak plasmas or its steady-state solution. The presented method minimizes calling the expensive theory-based transport code, while maintains high spatial resolution and robust convergence 


\section{Acknowledgement}

This work is based upon work supported by the US DOE, Office of Science, Office of Fusion Energy Sciences, using the DIII-D National Fusion Facility, a DOE Office of Science user facility, under awards, DE-AC05-00OR22725, DE-FG02-95ER54698, and DE-FG0295ER54309. 


\section{References}

[1] R.E. Waltz, G.M. Staebler, W. Dorland, G.W. Hammett, M. Kotschenreuther, and J.A. Konings, Phys. Plasmas 4, 2482 (1997).

[2] G. Bateman, A.H. Kritz, J.E. Kinsey, A.J. Redd, and J. Weiland, Phys. Plasmas 5, 1793 (1998).

[3] G.M. Staebler, J.E. Kinsey, and R.E. Waltz, Phys. Plasmas 14, 055909 (2007).

[4] S.P. Hirshman and D.J. Sigmar, Nucl. Fusion 21, 1079 (1981).

[5] H.E. St. John, T.S. Taylor, Y.R. Lin-Liu, and A.D. Turnbull, Proceedings of the 15th International Conference on Plasma Physics and Controlled Nuclear Fusion Research, Seville, Spain, 1994 IAEA, Vienna, 1995 , Vol. 3, p. 603.

[6] S.C. Jardin, G. Bateman, G.W. Hammett, L.P. Ku, Journal of Comput. Phys. 227, 8769 (2008).

[7] J. Candy, C. Holland, R.E. Waltz, M.R. Fahey, and E. Belli, Phys. Plasmas 16, 060704 (2009).

[8] J. Nocedal and S.J. Wright, Numerical Optimization, Springer-Verlag, New York, 1999.

[9] T. Aoki, Comput. Phys. Commun. 102, 132-146 (1997).

[10] W. Elwasif, D.E. Bernholdt, A.G. Shet, S.S. Foley, R. Bramley, D.B. Batchelor and L.A. Berry, Parallel, Distributed and Network-Based Processing (PDP), 2010 18th Euromicro International Conference on, 419-427 (2010).

[11] J.M. Park, E.J. Doyle, J.R. Ferron, C.T. Holcomb, G.L. Jackson, L.L. Lao, T.C. Luce, L.W. Owen, M. Murakami, T.H. Osborne, P.A. Politzer, R. Prater, and P.B. Snyder, Proc. of 23rd Int. Conf. on Fusion Energy, Paper EXC/P2-05, Daejeon, Korea (2010).

[12] J.R. Ferron, C.T. Holcomb, T.C. Luce, J.M. Park, P.A. Politzer, F. Turco, W.W. Heidbrink, E.J. Doyle, J.M. Hanson, A.W. Hyatt, Y. In, R.J. La Haye, M.J. Lanctot, M. Okabayashi, T.W. Petrie, C.C. Petty, and L. Zeng, Phys. Plasmas 20, 092504 (2013).

[13] C.T. Holcomb, J.R. Ferron, T.C. Luce, T.W. Petrie, J.M. Park, F. Turco, M.A. Van Zeeland, M. Okabayashi, C.T. Lasnier, J.M. Hanson, P.A. Politzter, Y. In, A.W. Hyatt, R.J. La Haye, and M.J. Lanctot, Nucl. Fusion 54, 093009 (2014). 
[14] Y.K.M. Peng, J.M. Canik, S.J. Diem, S.L. Milora, J.M. Park, A.C. Sontag, P.J. Fogarty, A. Lumsdaine, M. Murakami, T.W. Burgess, M.J. Cole, Y. Katoh, K. Korsah, B.D. Patton, J.C. Wagner, G.L. Yoder, R. Stambaugh, G. Staebler, M. Kotschenreuther, P. Valanju, S. Mahajan, and M. Sawan, Fusion Sci. Tech. 60, 441 (2011).

[15] M. Murakami, J.M. Park, G. Giruzzi, J. Garcia, P. Bonoli, R.V. Budny, E.J. Doyle, A. Fukuyama, N. Hayashi, M. Honda, A. Hubbard, S. Ide, F. Imbeaux, E.F. Jaeger, T.C. Luce, Y.-S. Na, T. Oikawa, T.H. Osborne, V. Parail, A. Polevoi, R. Prater, A.C.C. Sips, J. Snipes, H.E. St. John, P.B. Snyder, I. Voitsekhovitch, and ITPA/Integrated Operation Scenario Group, Nucl. Fusion 51, 103006 (2011).

[16] F. Wagner, A. Becoulet, R. Budny, V. Erckmann, D. Farina, G. Giruzzi, Y. Kamada, A. Kaye, F. Koechl, K. Lackner, N. Marushchenko, M. Murakami, T. Oikawa, V. Parail, J. M. Park, G. Ramponi, O. Sauter, D. Stork, P. R. Thomas, Q. M. Tran, D. Ward, H. Zohm, and C. Zucca, Plasma Phys. Control. Fusion 52, 124044 (2010).

[17] C.S. Chang and F. L. Hinton, Phys. Fluids 25, 1493 (1982).

[18] A. Pankin, D. McCune, R. Andre, G. Bateman, and A. Kritz, Comput. Phys. Commun. 159, 157 (2004).

[19] Y.R. Lin-Liu, V.S. Chan and R. Prater, Phys. Plasmas 10, 4064 (2003).

[20] L.L. Lao, J.R. Ferron, R.J. Groebner, W. Howl, H. St. John, E.J. Strait, and T.S. Taylor, Nucl. Fusion 30, 1035 (1990). 

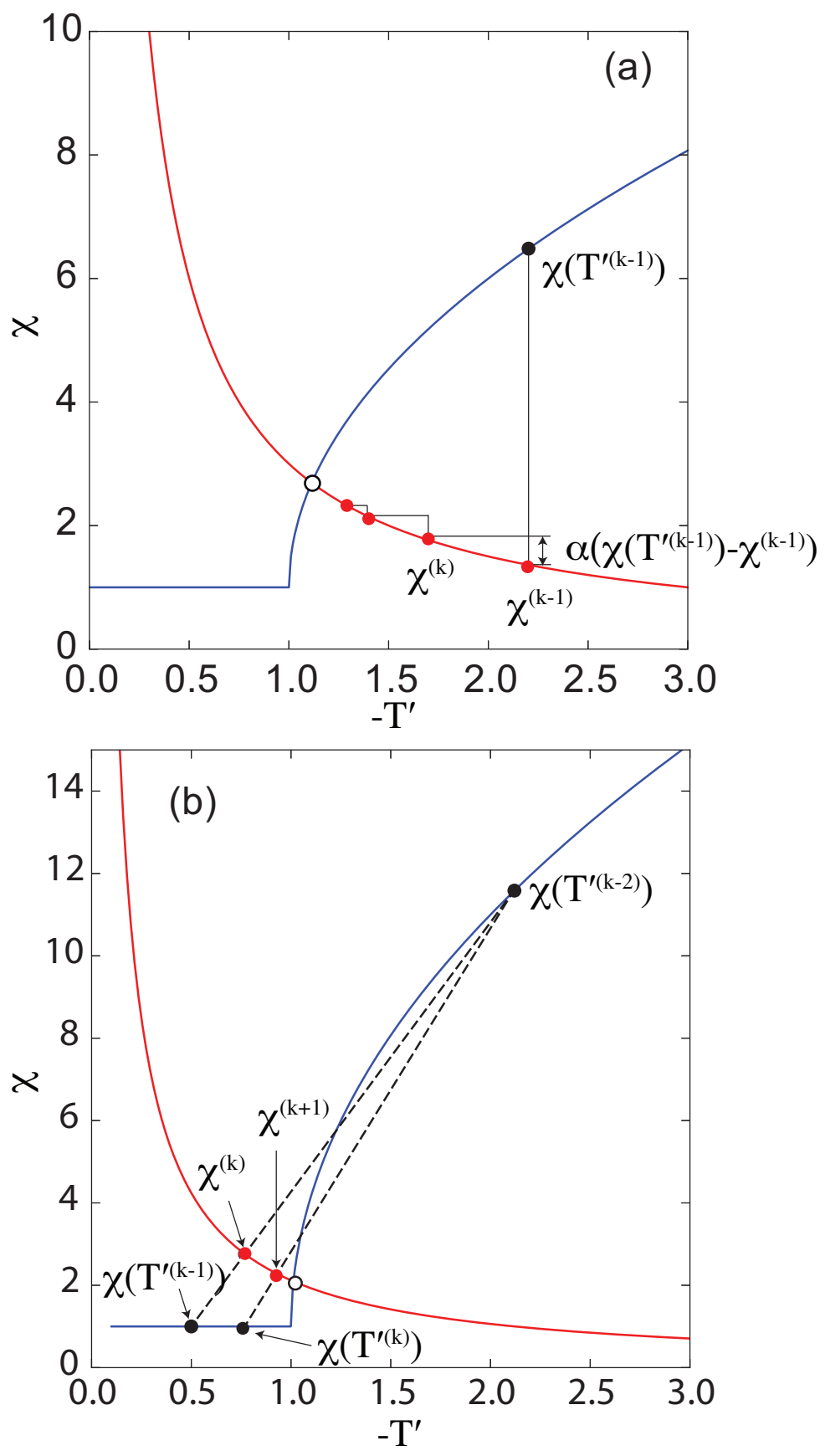

Figure 1: The $\chi^{k}$ (red circle) converges to the solution of $\chi\left(T^{\prime}\right) T^{\prime}=C$ (open circle), which is the intersection of $y=\chi\left(T^{\prime}\right)$ (blue line) and $y=C / T^{\prime}$ (red line). (a) under-relaxation method and (b) regula-falsi method. 


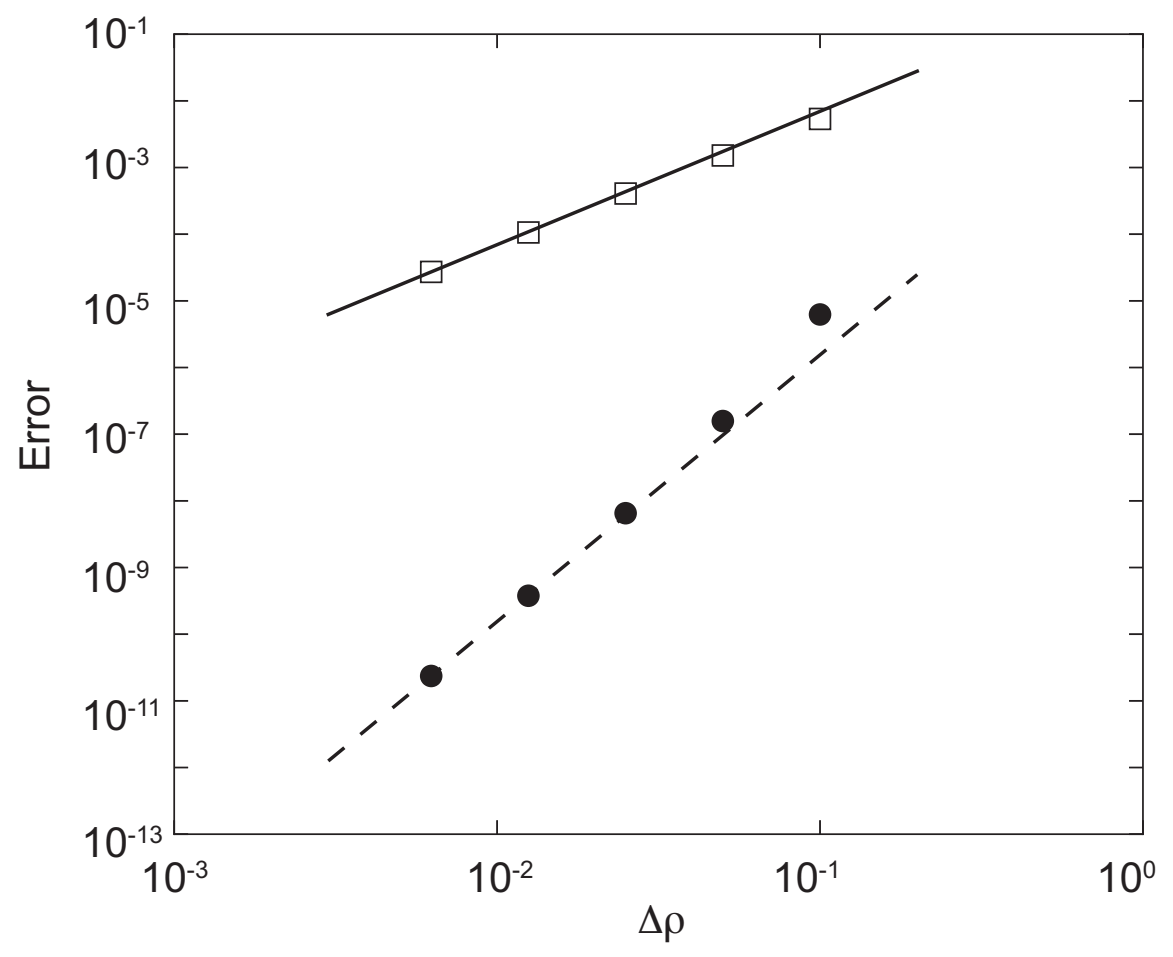

Figure 2: Comparison of numerical accuracy between IDO (circle) and 2nd order FDM (square) for different number of grid points. The solid (dashed) line corresponds to the 2nd (4th) order convergence. 

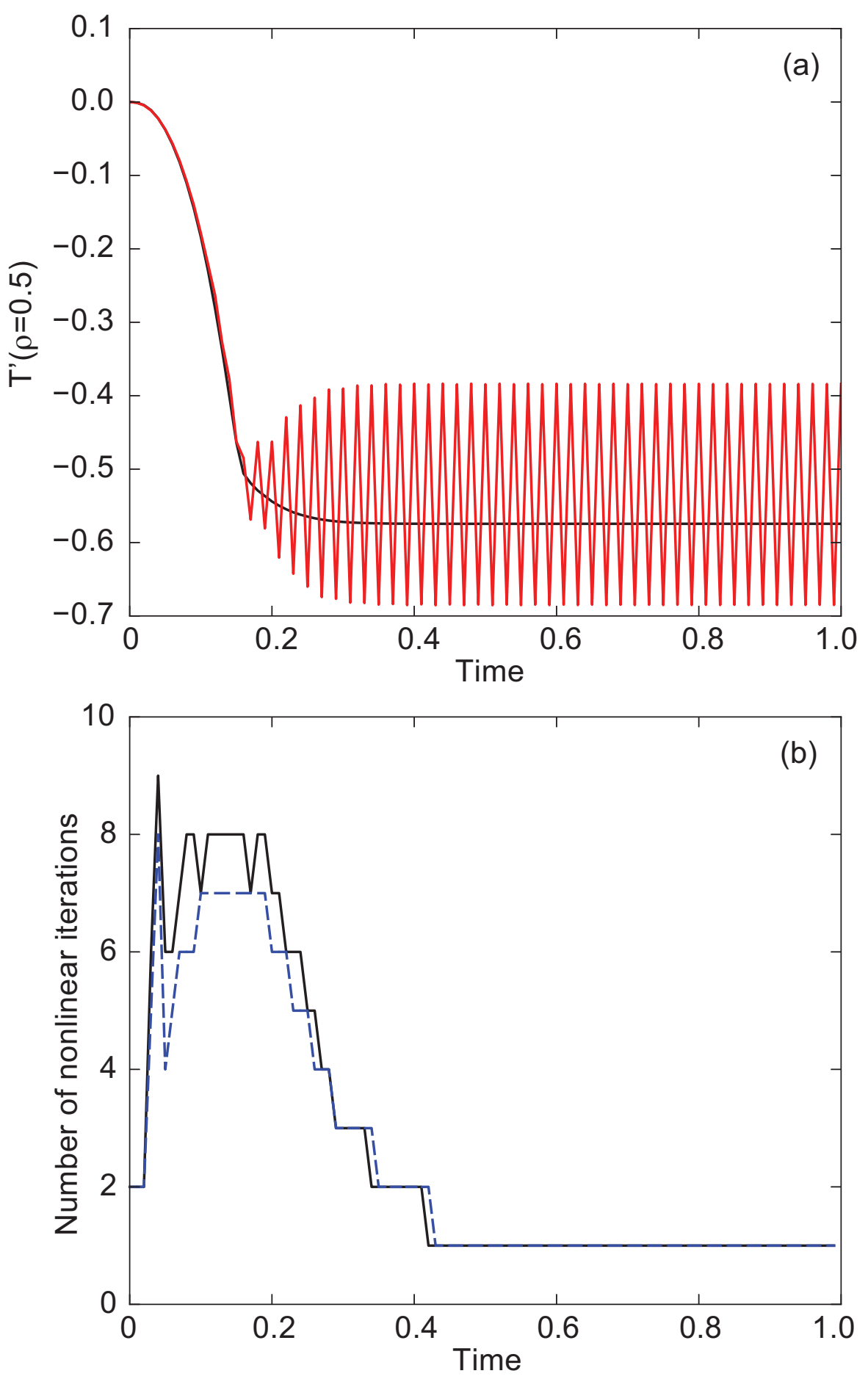

Figure 3: (a) Time trace of the calculated $T^{\prime}$ at the half minor radius for implicit (black) and explicit (red) time advance, (b) Number of nonlinear iterations for the under-relaxation (solid) and regula-falsi (dashed) methods, respectively. 

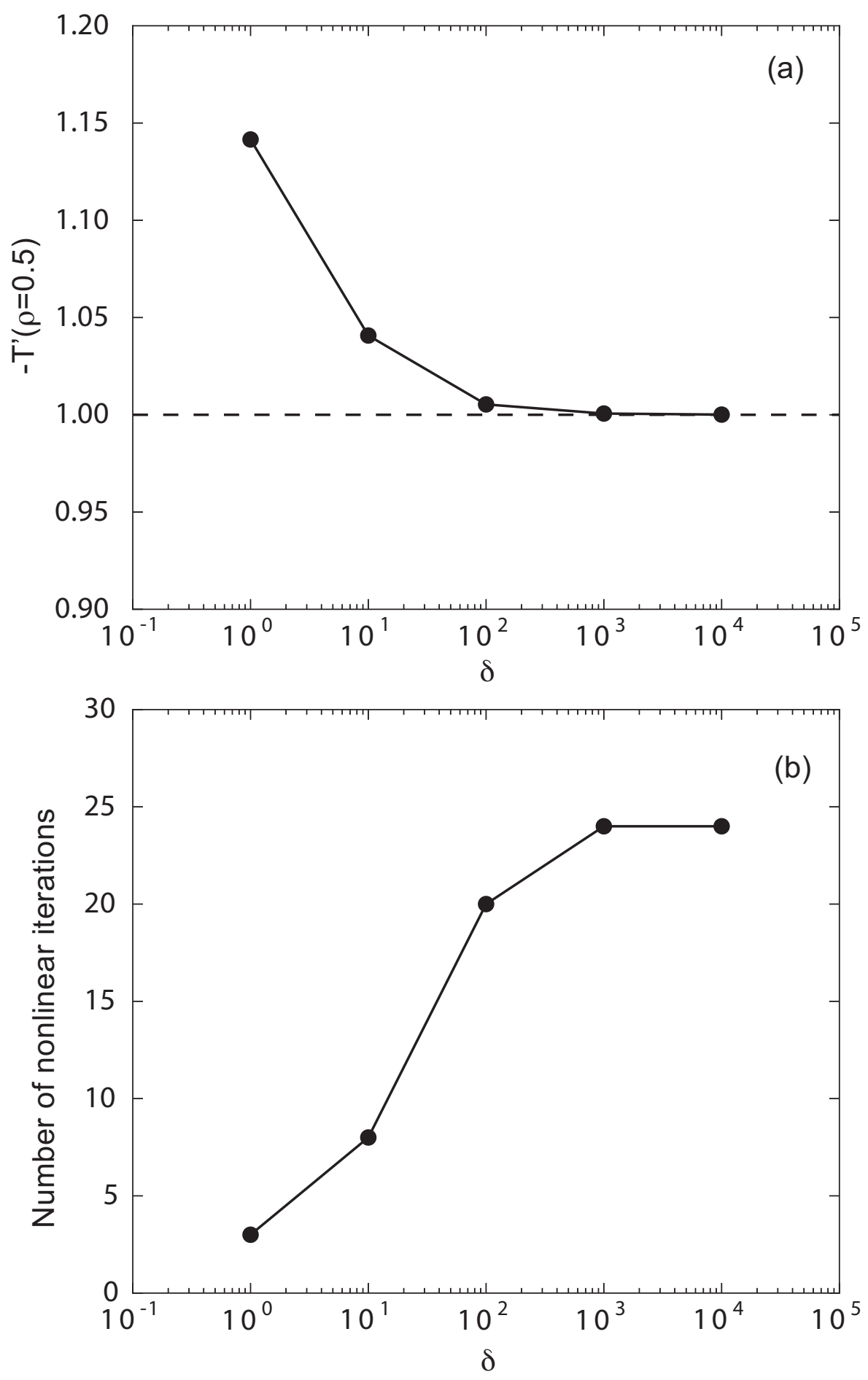

Figure 4: (a) Converged numerical solution of $-T^{\prime}(\rho=0.5)$ and (b) number of nonlinear iterations as a function of $\delta$. 

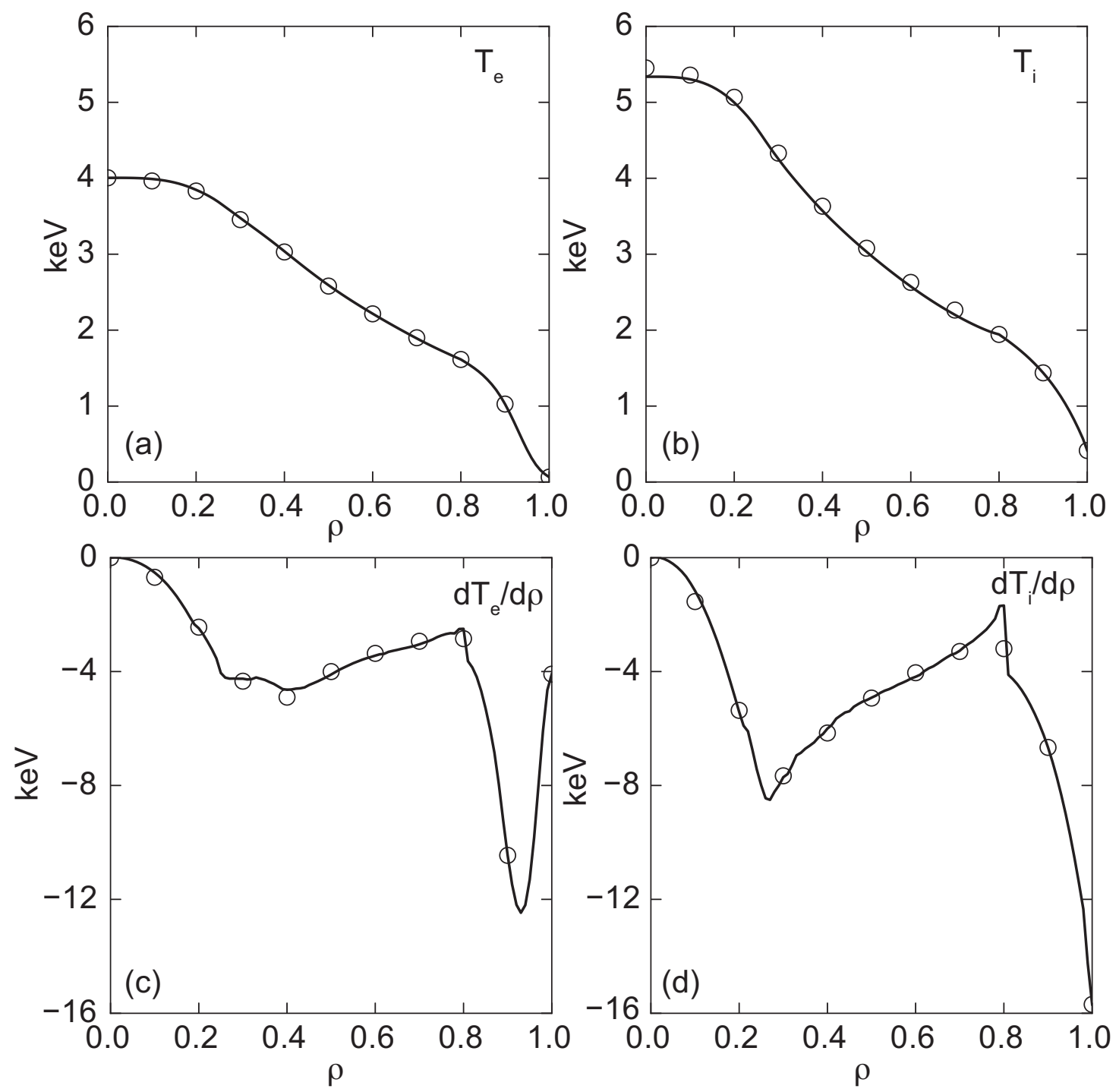

Figure 5: Calculated (a) $T_{e}$, (b) $T_{i}$, (c) $T_{e}^{\prime}$, and (d) $T_{i}^{\prime}$ profiles with TGLF for $N=101$ (line) and $N=11$ (symbol). 


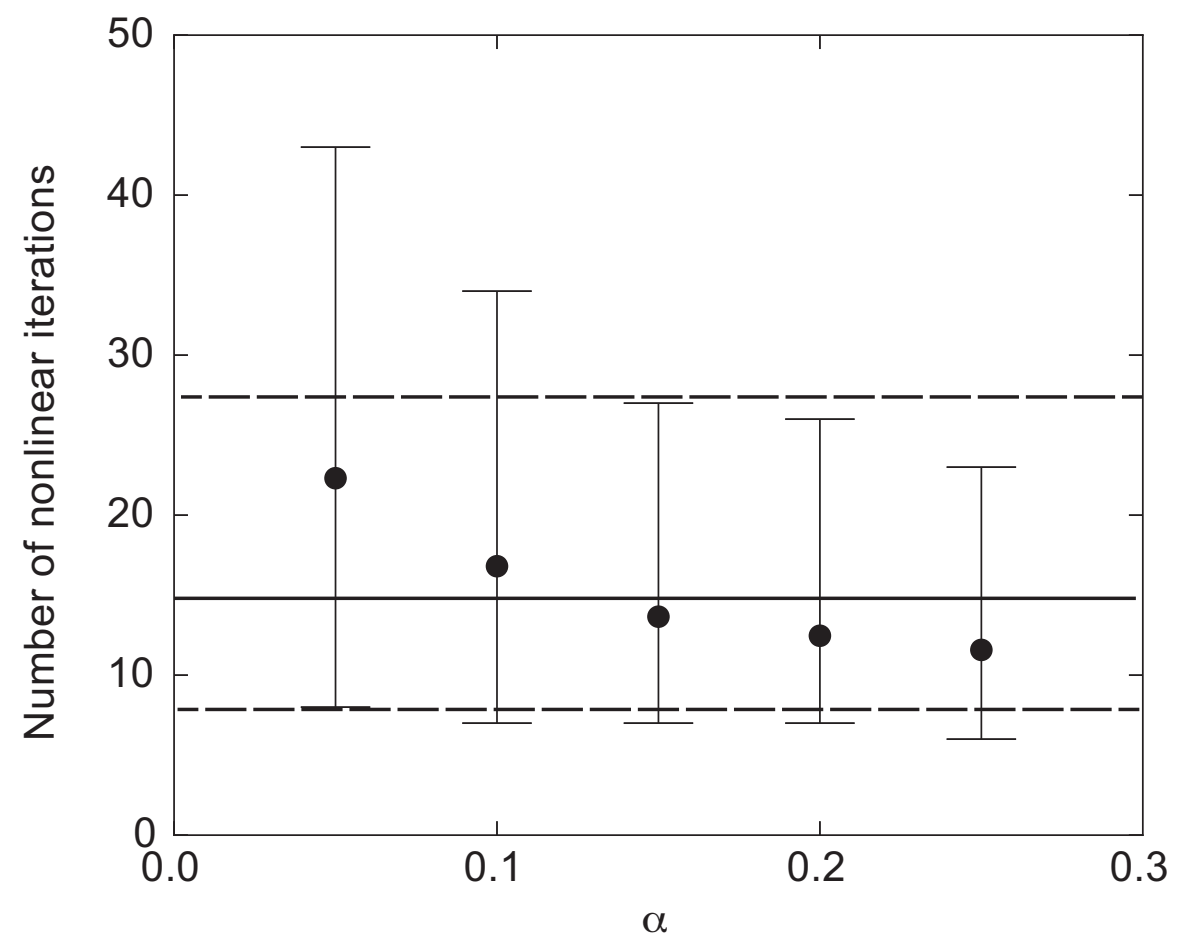

Figure 6: Average number of nonlinear iterations for steady-state solution of 27 DIII-D high beta discharges with the under-relaxation (symbol) and regula-falsi (solid line) methods. The error bar and dashed lines denote the maximum and minimum number of iterations. 